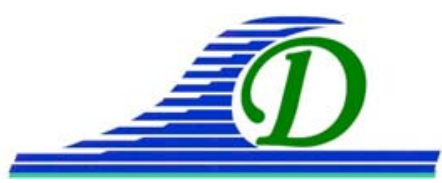

XIII ${ }^{\text {èmes }}$ Journées Nationales Génie Côtier - Génie Civil

Dunkerque, 2-4 juillet 2014

DOI:10.5150/jngcgc.2014.040 @ Editions Paralia CFL

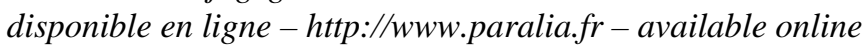

\title{
Prise en compte de l'hétérogénéité granulométrique du sédiment en morphodynamique côtière
}

\section{Thomas GUERIN ${ }^{1}$, Xavier BERTIN ${ }^{1}$, Eric CHAUMILLON ${ }^{1}$, Guillaume DODET ${ }^{1}$}

\section{Université de La Rochelle, UMR 6250 LIENSs CNRS,}

2 rue Olympe de Gouges, 17000 La Rochelle, France.

thomas.guerin@univ-Ir.fr ; xavier.bertin@univ-Ir.fr ; eric.chaumillon@univ-Ir.fr ; guillaume.dodet@univ-lr.fr

\begin{abstract}
Résumé :
Dans cet article nous montrons l'intérêt de prendre en compte l'hétérogénéité granulométrique naturelle d'un sédiment non-cohésif en modélisation morphodynamique côtière. Pour cela une approche "multi-classe multi-couche" a été utilisée, considérant le fond sédimentaire comme constitué de grains de sable de différentes tailles, ainsi que de plusieurs couches distinctes.

Une application au cas 1D de la migration d'une dune hydraulique puis au cas 2DH du banc de sable estuarien de la Longe de Boyard, situé à l'embouchure nord de la Baie de Marennes-Oléron, a mis en évidence la plus-value de cette approche par rapport à celle se limitant à un sédiment complètement homogène. Tout d'abord une modification des vitesses de migration est obtenue, celles-ci étant significativement plus faibles lorsqu'un sédiment hétérogène est pris en compte. Enfin, l'impact de différents forçages sur la distribution granulométrique surfacique et verticale du sédiment a pu être étudié grâce à cette approche.
\end{abstract}

Mots-clés : Modélisation morphodynamique, Sédiment non-cohésif, Hétérogénéité granulométrique du sédiment, Banc tidal, Stratigraphie.

\section{Introduction}

Les environnements côtiers sableux mixtes, soumis aux actions combinées des vagues et de la marée se caractérisent à la fois par une très forte dynamique et des enjeux socioéconomiques importants. La compréhension et la prédiction de leurs évolutions morphologiques représente un vrai défi dans le domaine de la morphodynamique côtière, du fait des différentes échelles spatiales et temporelles intervenant, de la nature variable du sédiment, du grand nombre de paramètres mis en jeu, ainsi que de la complexité des processus physiques et de leurs interactions. L'utilisation d'hypothèses simplificatrices en modélisation morphodynamique est alors nécessaire, et parmi cellesci la considération d'un sédiment totalement homogène est souvent retenue. Dans cet article nous montrons cependant que la prise en compte de l'hétérogénéité granulométrique d’un sédiment non-cohésif par une approche "multi-classe multi- 


\section{Thème 2 - Dynamique sédimentaire}

couche" peut être bénéfique sur deux points: (1) la modification des vitesses de migration des corps sédimentaires étudiés, et (2) la mise en relation possible entre la variation granulométrique du sédiment et des données stratigraphiques.

Après une description du système de modélisation morphodynamique et de l'approche multi-classe multi-couche utilisée, nous présentons les résultats de son application au cas 1D de la migration d'une dune hydraulique dans un canal, puis au cas 2DH de l'évolution du banc de sable estuarien de la Longe de Boyard situé à l'entrée nord de la Baie de Marennes-Oléron et soumis à différents forçages.

\section{Matériel et méthodes}

\subsection{Le système de modélisation morphodynamique}

Le système de modélisation utilisé réalise le couplage complet entre le code de circulation hydrodynamique SELFE (ZHANG \& BAPTISTA, 2008), le modèle spectral d'état de mer WWMII (ROLAND et al., 2012) et le module de transport sédimentaire SED2D (DODET, 2014), ces différents modules partageant les mêmes grilles nonstructurées et décomposition de domaine. L'approche multi-classe multi-couche (décrite dans la section suivante) a été intégrée au module SED2D, ce dernier résolvant l'équation de conservation du sédiment (équation d'Exner) afin de calculer l'évolution du fond à chaque pas de temps :

$\frac{\partial z_{b}}{\partial t}+\frac{1}{1-\lambda} \nabla q_{t o t}=0$

avec $z_{b}$ la côte du fond, $\lambda$ la porosité du sédiment, et $q_{\text {tot }}$ le transport sédimentaire total en $\mathrm{m}^{2} \mathrm{~s}^{-1}$.

\subsection{Description de l'approche multi-classe multi-couche}

Afin de modéliser l'évolution de la distribution horizontale et verticale du diamètre moyen du sédiment, le fond sédimentaire est considéré comme étant constitué de $\mathrm{N}$ classes et $\mathrm{M}$ couches de sédiment superposées les unes en dessous des autres. Chaque classe est définie par un diamètre médian $\left(d_{50}\right)$ et une fraction correspondante (la somme des fractions étant égale à 1), tandis que chaque couche est caractérisée par une épaisseur donnée. Notre approche est basée sur les travaux de RENIERS et al. (2013), où seule la couche de sub-surface a une épaisseur variable dans le temps.

Dans le cas d'un dépôt, les fractions au sein des couches de surface $\left(F_{1}\right)$ et de subsurface $\left(F_{2}\right)$ évoluent de la manière suivante :

$F_{1}(i, t+\Delta t)=\frac{\left(h_{t o p}+\Delta z_{t o t}\right) F_{1}(i, t)-\Delta z(i)}{h_{\text {top }}}$
$F_{2}(i, t+\Delta t)=\frac{h_{\text {sub }} F_{2}(i, t)-\Delta z_{\text {tot }} F_{1}(i, t)}{h_{\text {sub }}-\Delta z_{\text {tot }}}$ 


\section{XIII ${ }^{\text {èmes }}$ Journées Nationales Génie Côtier - Génie Civil \\ Dunkerque, 2-4 juillet 2014}

avec $i$ la classe de sédiment, $\Delta t$ le pas de temps, $h_{\text {top }}$ et $h_{\text {sub }}$ les épaisseurs respectives des couches de surface et sub-surface, $\Delta z(i)$ la variation de la côte du fond pour la classe $i$, et $\Delta z_{\text {tot }}$ la variation totale de celle-ci (somme des $\left.\Delta z(i)\right)$ qui est négatif dans ce cas.

Lors d'une érosion ( $\Delta z_{\text {tot }}$ positif), seules les fractions correspondant à la couche de surface varient dans un premier temps :

$F_{1}(i, t+\Delta t)=\frac{h_{t o p} F_{1}(i, t)+\Delta z_{t o t} F_{2}(i, t)-\Delta z(i)}{h_{\text {top }}}$

Cependant, pour éviter que l'épaisseur de la couche de sub-surface ne devienne trop petite ou trop importante, celle-ci est divisée en deux si $h_{\text {sub }}$ dépasse une valeur critique maximale, ou fusionne avec la couche inférieure si $h_{\text {sub }}$ devient plus petit qu'une valeur critique minimale. Suivant le cas, soit la dernière couche est supprimée, soit une couche supplémentaire est ajoutée afin de conserver un nombre constant de couches, les fractions correspondantes étant alors recalculées.

Le transport total est alors égal à la somme des transports de chaque classe, pondérés par leur fraction respective.

\section{Résultats}

\subsection{Cas 1D de la migration d'une dune hydraulique}

La migration d'une dune hydraulique dans un chenal sous l'influence d'un débit constant est modélisée numériquement en résolvant l'équation de conservation du sédiment en 1D avec un schéma de différences finies explicite et centré en espace. Le transport sédimentaire total $\left(q_{t o t}\right)$, somme des transports par charriage et par suspension, est calculé avec la formule empirique simplifiée de VAN RIJN (2007). L'effet de pente sur le transport total est également pris en compte en lui ajoutant un terme proportionnel à la pente locale (LARSON et al., 2011).

Les résultats pour un sédiment homogène de $d_{50}$ égal à $0.5 \mathrm{~mm}$ et pour un sédiment hétérogène à 5 classes $\left(0.1,0.35,0.5,1\right.$, et $1.8 \mathrm{~mm} ; d_{50}$ moyen égal à $0.5 \mathrm{~mm}$; fractions initiales homogènes égales à 0.2 ), et 10 couches, sont comparés après une simulation de $10 \mathrm{~h}$ (fig. 1). Le pas de temps est d'une minute, la discrétisation spatiale de $25 \mathrm{~m}$, et le courant maximal est d'environ $1.7 \mathrm{~m} \mathrm{~s}^{-1}$ au niveau de la crête de la dune au début de la simulation, la condition CFL étant satisfaite. La figure 2 montre la répartition du $d_{50}$ moyen du sédiment hétérogène au sein des différentes couches à la fin de la simulation.

On constate tout d'abord que l'approche multi-classe multi-couche présente des oscillations numériques d'amplitude réduite par rapport au cas d'un sédiment homogène, ces oscillations provenant de la nature non-linéaire du couplage entre le transport sédimentaire et l'évolution du fond. Cette évolution du fond étant proportionnelle à la divergence du flux sédimentaire, la diminution des oscillations numériques s'explique par la diminution du fort gradient de transport (en valeur absolue) présent au point situé juste en aval de la crête de la dune. La vitesse de migration de la dune est ainsi 


\section{Thème 2 - Dynamique sédimentaire}

diminuée, celle-ci étant proportionnelle au gradient de transport. Plus précisément, soit i ce point où $\left|\nabla\left(q_{t o t}(i)\right)\right|$ est maximale, le sédiment hétérogène devenu plus grossier au point $\mathrm{i}-1$ et plus fin au point $\mathrm{i}+1$ va respectivement diminuer le transport en i-1 et l'augmenter en i+1, par rapport au cas du sédiment homogène. En effet pour un courant relativement fort comme c'est le cas dans cet exemple, le transport du sédiment fin, essentiellement par suspension, prédomine largement celui du sédiment grossier (SOULSBY, 1997). Une répartition de taille de grain caractéristique des dunes sousmarines apparaît alors, avec du sédiment plus grossier au niveau de la zone en érosion (sur la crête et la pente de la dune faisant face au courant), et du sédiment plus fin au niveau de la zone en accrétion (sur le creux et la pente de la dune en aval du courant) (WALGREEN et al., 2003, 2004 ; HOLLAND \& ELMORE, 2008).
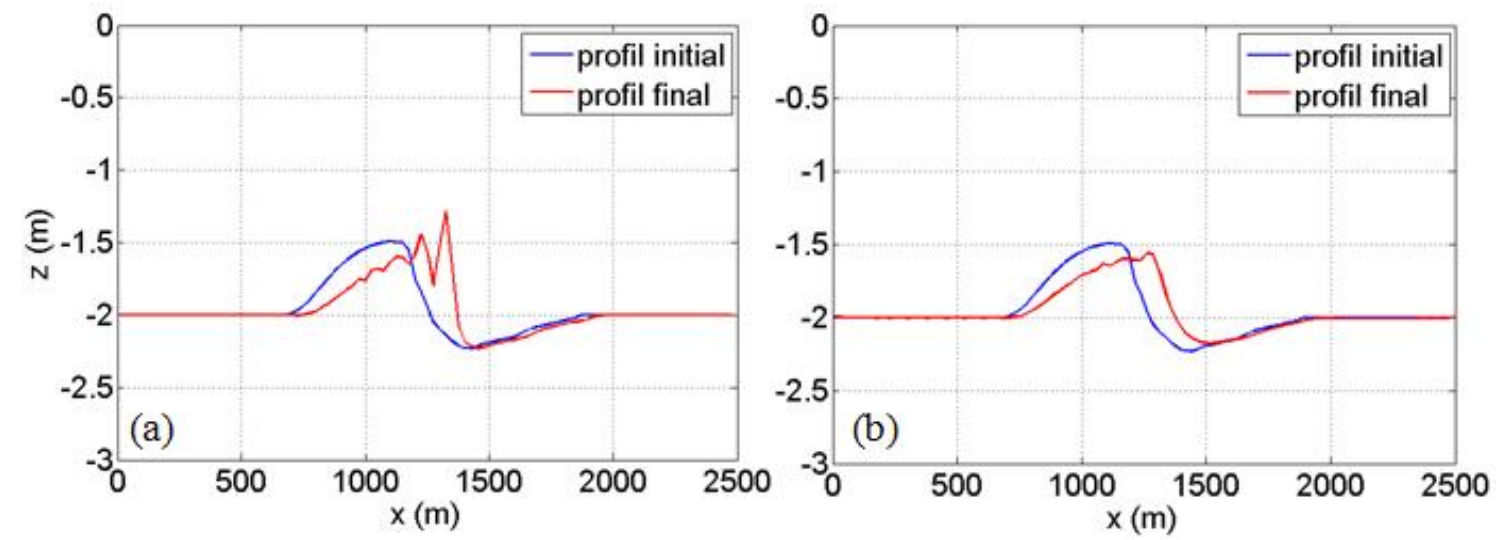

Figure 1. Migration d'une dune hydraulique après $10 \mathrm{~h}$ de simulation.

(a) Sédiment homogène ( $d_{50}=0.5 \mathrm{~mm}$ ). (b) Sédiment hétérogène (5 classes, 10 couches, $d_{50 \text { moy }}=0.5 \mathrm{~mm}$ ).

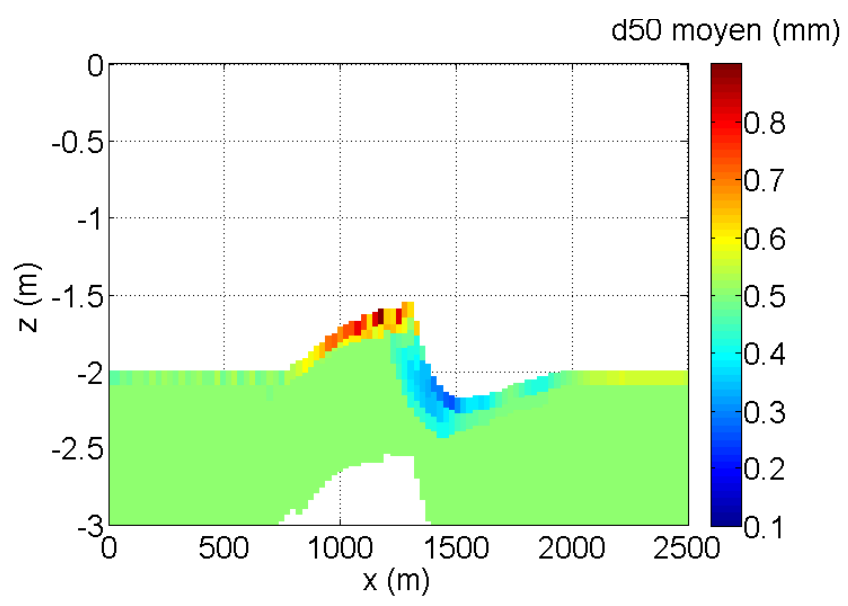

Figure 2. Distribution du $d_{50}$ moyen après $10 \mathrm{~h}$ de simulation dans le cas d'un sédiment hétérogène. 


\section{XIII ${ }^{\text {èmes }}$ Journées Nationales Génie Côtier - Génie Civil \\ Dunkerque, 2-4 juillet 2014}

\subsection{Cas de la Longe de Boyard}

La Longe de Boyard est un banc de sable estuarien d'environ $8 \mathrm{~km}$ par $2 \mathrm{~km}$ situé à l'embouchure nord de la Baie de Marennes-Oléron, entre l'île d'Oléron et l'île d'Aix (figure 3). Il est orienté suivant l'axe nord-ouest sud-est correspondant à la direction principale des courants de marée dans cette zone (BERTIN \& CHAUMILLON, 2005). La crête de ce banc est peu profonde puisqu'elle est localement émergée lors des marées basses de vives-eaux, et les deux chenaux présents sur ses flancs atteignent environ 17 m Cote Marine. Son extrémité nord est caractérisée par une pente plus douce que l'extrémité sud, tandis que ses flancs présentent des pentes maximales de l'ordre de 0.03 à $0.04 \mathrm{~m} \cdot \mathrm{m}^{-1}$. Une asymétrie est observée concernant les courants de marée résiduels, la Longe étant dominée par le flot sur son flanc est et par le jusant sur son flanc ouest (TESSON, 1973), ceci créant une circulation résiduelle horaire autour du banc, commune à ce type de corps sédimentaire (DYER \& HUNTLEY, 1999 ; ZIMMERMAN, 1981). Bien que la zone de la Longe soit géographiquement abritée du large par l'île d'Oléron, l'action de la houle y est relativement importante du fait d'une forte réfraction convexe sur le toit du banc (hauteurs significatives de l'ordre de $50 \%$ par rapport au large). Enfin, la Longe est également soumise à l'action importante des mers de vent en raison des faibles profondeurs.

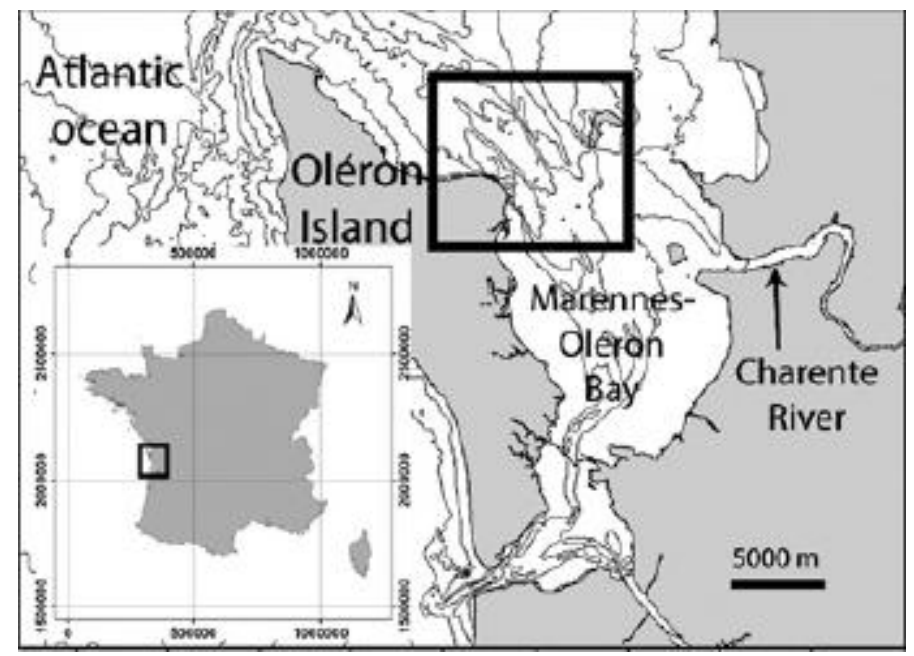

Figure 3. Localisation du banc de sable estuarien la Longe de Boyard.

Grâce au modèle, l'évolution morphologique de la Longe de Boyard est simulée sur une période de 2 ans avec la marée pour seul forçage, définie par ses 19 constituants principaux. Comme dans le cas 1D de la dune hydraulique, la formule de transport sédimentaire utilisée est celle de VAN RIJN (2007). La figure 4 (c) et (d) montre une comparaison entre la bathymétrie finale obtenue au niveau de l'extrémité sud du banc, zone où le transport sédimentaire résiduel est relativement fort (voir fig. 5 (a)), dans le cas d'un sédiment homogène ( $d_{50}$ de $\left.0.5 \mathrm{~mm}\right)$ et dans le cas d'un sédiment hétérogène 


\section{Thème 2 - Dynamique sédimentaire}

(10 classes, 10 couches, $d_{50}$ moyen de $0.5 \mathrm{~mm}$ ). On constate, en accord avec la partie 3.1., que la prise en compte d'un sédiment hétérogène diminue la vitesse de migration de cette partie du banc, réduisant par ailleurs l'amplitude des oscillations numériques. Un résultat similaire est obtenu au niveau de la partie nord du banc (non montré).
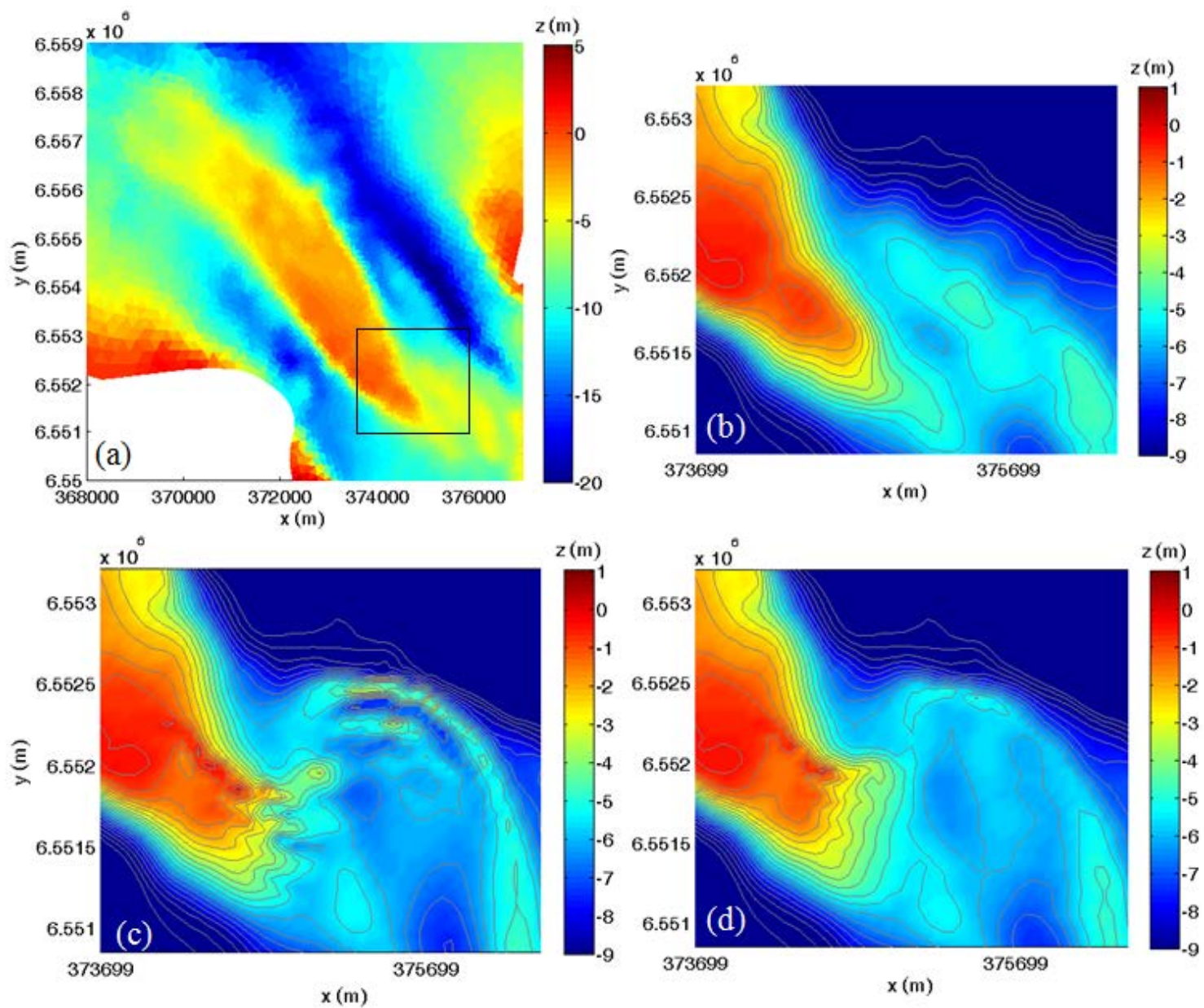

Figure 4. (a) Bathymétrie de la Longe de Boyard de 1824. (b) Zoom sur l'extrémité sud de la Longe. (c) et (d) Bathymétrie après 2 ans de simulation : dans le cas d'un sédiment homogène $(c)$ et dans le cas d'un sédiment hétérogène $(d)$.

Cependant, si on compare les simulations avec les observations à plus long terme, on constate que l'on simule un déplacement vers le nord-est des extrémités nord et sud de la Longe, ce qui concorde avec la tendance à long terme observée pour l'extrémité sud du banc mais contredit celle concernant l'extrémité nord (CHAUMILLON et al., 2008). Afin d'expliquer ce comportement, nous comparons une simulation forcée seulement par la marée avec une simulation forcée par la marée en présence d'un climat de vagues énergétique représentatif de conditions d'agitation hivernales (3 m de hauteur significative, 12 s de période pic, et de direction N290 à la frontière), afin de bien 


\section{XIII ${ }^{\text {èmes }}$ Journées Nationales Génie Côtier - Génie Civil \\ Dunkerque, 2-4 juillet 2014}

distinguer l'impact des vagues sur le transport sédimentaire dans cette zone. Le caractère asymétrique de la houle est pris en compte pour ce second forçage, selon la paramétrisation de ELFRINK et al. (2006). La formule de CAMENEN \& LARSON (2005, 2008) est alors utilisée pour le calcul du transport total, car elle permet d'intégrer l'effet de cette asymétrie sur le transport.

En comparant les deux cartes de transport sédimentaire résiduel correspondant à une marée de vives-eaux (fig. 5), on constate tout d'abord que la présence de vagues diminue fortement l'intensité et le sens de ce transport résiduel. En effet, celui-ci étant globalement dirigé vers l'extérieur de la baie dans le cas du forçage par la marée, la présence des vagues entraîne un inversement de cette tendance sur une majeure partie de la zone. Les courants induits par les vagues sur le banc étant négligeables, cet impact sur la direction du transport est directement dû au caractère asymétrique des vagues puisque considérer une houle symétrique modifie seulement l'intensité du transport calculé par la formule et non sa direction, par rapport au cas sans vagues.

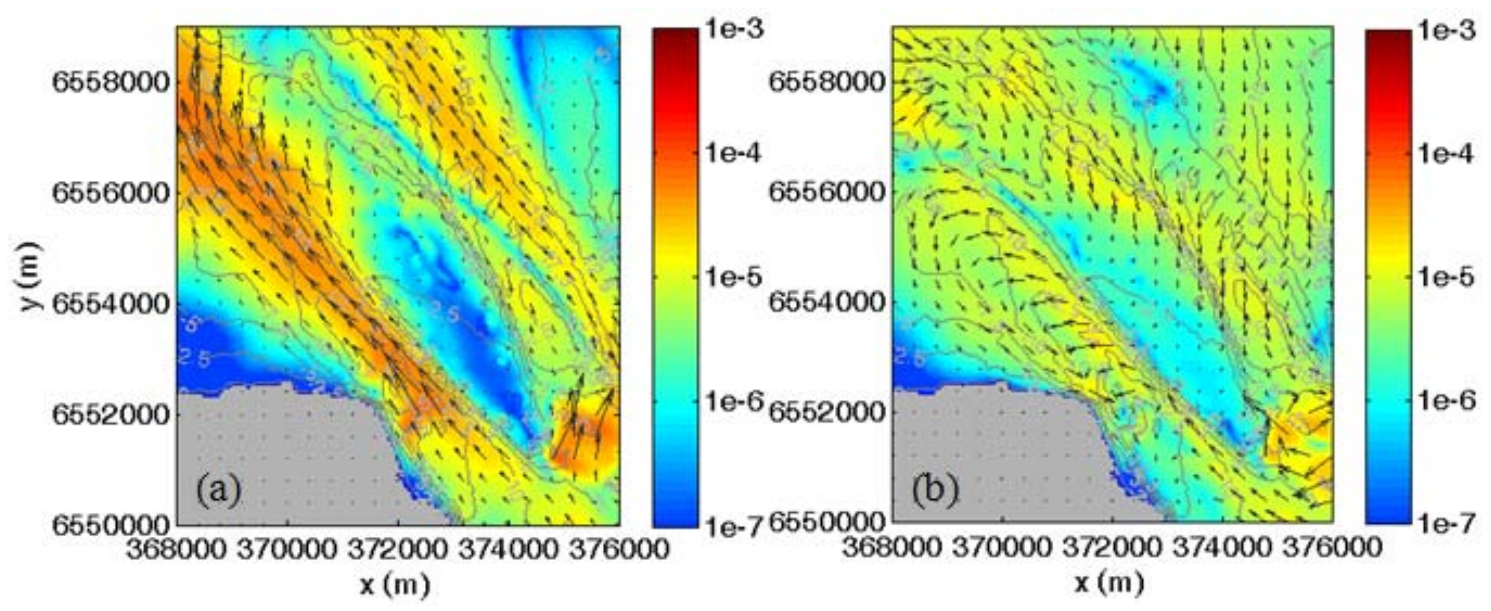

Figure 5. Transport total résiduel $\left(\mathrm{m}^{2} \mathrm{~s}^{-1}\right)$ au nord de la Longe, calculé sur un cycle de vives-eaux. (a) Forçage par la marée. (b) Forçage par la marée et une houle modérée au large ( $H s=3 \mathrm{~m}, \mathrm{Tp}=12 \mathrm{~s}$ ).

Comme dans le cas précédent de la dune hydraulique, le lien direct entre le transport résiduel et la variation granulométrique du sédiment est mis en évidence par l'utilisation de l'approche multi-classe multi-couche. La figure 6 montre la distribution du $d_{50}$ moyen du sédiment le long d'un profil situé sur la partie nord du banc, après 5 jours de simulation et pour les deux types de forçages (marée seule et marée avec vagues). En accord avec la partie 3.1., on observe du sédiment plus grossier en zone d'érosion, et plus fin en zone de dépôt. Un résultat important est l'inversion de cette répartition entre les deux forçages sur le côté ouest du profil (fig. 6 (b) et (c)), due à l'inversion de la tendance érosion/dépôt dans cette zone. La forte fluctuation saisonnière des hauteurs significatives dans l'Atlantique nord-est (DODET et al., 2010) pourrait donc entrainer 


\section{Thème 2 - Dynamique sédimentaire}

l'alternance de dépôts de sables fins et grossiers au niveau des flancs de la Longe de Boyard. Les discontinuités (ou réflecteurs) observées sur les profils sismiques présentés par CHAUMILLON et al. (2008) pourraient donc correspondre à un enregistrement sédimentaire du cycle saisonnier des hauteurs de vague. En effet ces réflecteurs, obtenus par prospection sismique, sont le plus souvent dus à une variation granulométrique au sein de la colonne sédimentaire (BILLEAUD et al., 2005).
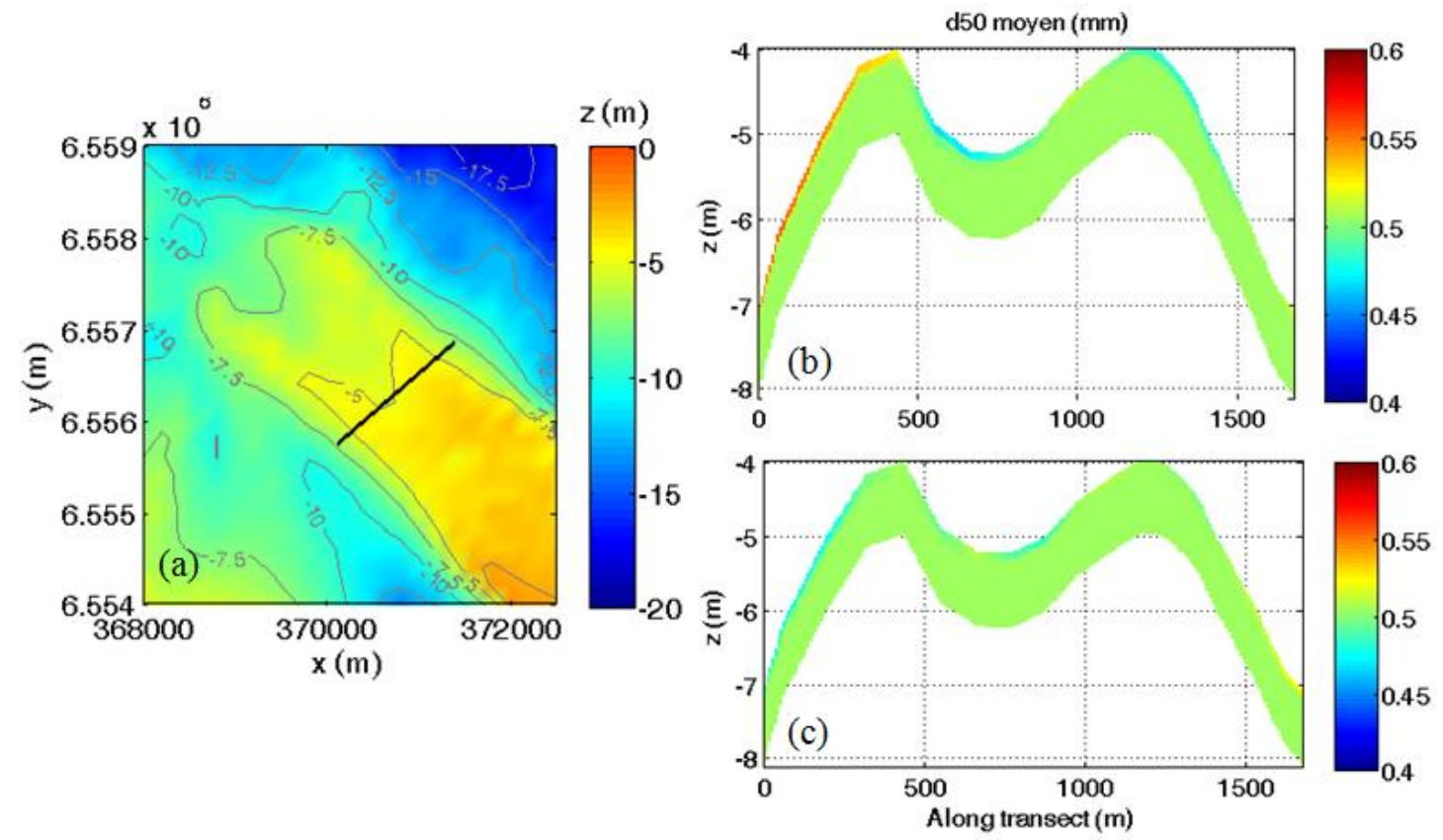

Figure 6. Distribution du $d_{50}$ moyen le long d'un profil au nord de la Longe.

(a) Localisation du profil. (b) Forçage par la marée. (c) Forçage par la marée et une houle modérée au large ( $\mathrm{Hs}=3 \mathrm{~m}, \mathrm{Tp}=12 \mathrm{~s})$.

\section{Conclusions}

Dans cette étude, la prise en compte de l'hétérogénéité granulométrique d'un sédiment non-cohésif en morphodynamique côtière est comparée à une approche plus classique considérant un sédiment homogène. Cette hétérogénéité est étudiée au moyen d'une approche multi-classe multi-couche basée sur les travaux de RENIERS et al. (2013). La première conséquence de l'utilisation de cette approche par rapport au cas d'un sédiment à granularité constante est la diminution des vitesses de migration (ou vitesses d'évolution du fond) au cours des simulations, dans un cas simple de migration d'une dune hydraulique en 1D sous l'action d'un courant stationnaire, ainsi que dans celui de l'évolution d'un banc de sable estuarien modélisée en 2DH et soumis à l'action de la marée. Le second résultat est la possibilité d'étudier les impacts respectifs de la marée et des vagues sur la distribution granulométrique surfacique et verticale du sédiment, et ainsi de comparer des profils sédimentaires synthétiques avec des profils stratigraphiques obtenus par géophysique. 


\section{XIII ${ }^{\text {èmes }}$ Journées Nationales Génie Côtier - Génie Civil \\ Dunkerque, 2-4 juillet 2014}

Bien que préliminaire, l'application de l'approche multi-classe multi-couche présentée ici ouvre de nombreuses perspectives pour la modélisation morphodynamique côtière et son couplage avec la stratigraphie.

\section{Références bibliographiques}

BERTIN X., CHAUMILLON E. (2005). Apports de la modélisation sur bathymétries historiques dans la compréhension des évolutions des bancs de sable estuariens. CR Géoscience, Vol. 337, pp 1375-1383. http://dx.doi.org/10.1016/j.crte.2005.06.007

BILLEAUD I., CHAUMILLON E., WEBER O. (2005). Evidence of a major environmental change recorded in a macrotidal bay (Marennes-Oléron Bay, France) by correlation between VHR seismic profiles and cores. Geo-Marine Letters, Vol. 25, pp 110. http://dx.doi.org/10.1007/s00367-004-0183-0

CAMENEN B., LARSON M. (2005). A general formula for non-cohesive bed load sediment transport. Estuarine, Coastal and Shelf Science, Vol. 63, pp 249-260. http://dx.doi.org/10.1016/j.ecss.2004.10.019

CAMENEN B., LARSON M. (2008). A general formula for noncohesive suspended sediment transport. Journal of Coastal Research, Vol. 24, pp 615-627. http://dx.doi.org/10.2112/06-0694

CHAUMILLON E., BERTIN X., FALCHETTO H., ALLARD J., WEBER N., WALKER P., POUVREAU N., WOPPELMANN G. (2008). Multi time-scale evolution of a wide estuary linear sandbank, the Longe de Boyard, on the French Atlantic coast. Marine Geology, Vol. 251, pp 209-223. http://dx.doi.org/10.1016/j.margeo.2008.02.014

DODET G., BERTIN X., TABORDA R. (2010). Wave climate variability in the NorthEast Atlantic Ocean over the last six decades. Ocean Modelling, Vol. 31, pp120-131. http://dx.doi.org/10.1016/j.ocemod.2009.10.010

DODET G. (2014). Morphodynamic modeling of wave-dominated tidal inlets. Thèse Université de La Rochelle.

DYER K.R., HUNTLEY D.A. (1999). The origin, classification and modeling of sand banks and ridges. Continental Shelf Research, Vol. 19, pp 1285-1330. http://dx.doi.org/10.1016/S0278-4343(99)00028-X

ELFRINK B., HANES D.M., RUESSINK B.G. (2006). Parametrization and simulation of near bed orbital velocities under irregular waves in shallow water. Coastal Engineering, Vol. 53, pp 915-927. http://dx.doi.org/10.1016/j.coastaleng.2006.06.002

HOLLAND K.T., ELMORE P.A. (2008). A review of heterogeneous sediments in coastal environments. Earth-Science Reviews, Vol. 89, pp 116-134. http://dx.doi.org/10.1016/j.earscirev.2008.03.003

LARSON M., CAMENEN B., NAM P.T. (2011). A unified sediment transport model for inlet application. Journal of Coastal Research, Vol. 59, pp 27-38. doi:10.2112/SI59-004.1 
RENIERS A.J.H.M., GALLAGHER E.L., MACMAHAN J.H., BROWN J.A., VAN ROOIJEN A.A., VAN THIEL DE VRIES J.S.M., VAN PROOIJEN B.C. (2013). Observations and modeling of steep-beach grain-size variability. Journal of Geophysical Research, Vol. 118, pp 1-15. http://dx.doi.org/10.1029/2012JC008073

ROLAND A., ZHANG Y.J., WANG H.V., MENG Y., TENG Y-C., MADERICH V., BROVCHENKO I., DUTOUR-SIKIRIC M., ZANKE U. (2012). A fully coupled 3D wave-current interaction model on unstructured grids. Journal of Geophysical Research, Vol. 117, C00J33. http://dx.doi.org/10.1029/2012JC007952

SOULSBY R. (1997). Dynamics of marine sands. Thomas Telford, London, 249 pp.

TESSON M. (1973). Aspects dynamiques de la sédimentation dans la baie de Marennes-Oléron (France). Thèse Université de Bordeaux, 128 pp.

VAN RIJN L.C. (2007). Unified view of sediment transport by currents and waves. I : Initiation of motion, bed roughness, and bed-load transport. Journal of Hydraulic Engineering, Vol. 133, pp 649-667. http://dx.doi.org/10.1061/ASCE0733-94292007133:6649

VAN RIJN L.C. (2007). Unified view of sediment transport by currents and waves. II : Suspended transport. Journal of Hydraulic Engineering, Vol. 133, pp 668-689. http://dx.doi.org/10.1061/ASCE0733-94292007133:6668

WALGREEN M., DE SWART H.E. (2003). Effect of grain size sorting on the formation of shoreface-connected sand ridges. Journal of Geophysical Research, Vol. 108, pp 8.1-8.16. http://dx.doi.org/10.1029/2002JC001435

WALGREEN M., DE SWART H.E., CALVETE D. (2004). A model for grain-size sorting over tidal sand ridges. Ocean Dynamics, Vol. 54, pp 374-384. http://dx.doi.org/10.1007/s10236-003-0066-3

ZHANG Y., BAPTISTA A.M. (2008). SELFE: A semi-implicit Eulerian-Lagrangian finite-element model for cross-scale ocean circulation. Ocean Modelling, Vol. 21, pp 71-96. http://dx.doi.org/10.1016/j.ocemod.2007.11.005

ZIMMERMAN J.T.F. (1981). Dynamics, diffusion and geomorphological significance of tidal residual eddies. Nature, Vol. 290, pp 549-555. http://dx.doi.org/10.1038/290549a0 